\title{
Degenerate sectors of the Ashtekar gravity
}

\author{
Jerzy Lewandowski and Jacek Wiśniewski \\ Instytut Fizyki Teoretycznej, Wydział Fizyki, Uniwersytet Warszawski, ul. Hoża 69, 00-681, \\ Warszawa, Poland
}

Received 3 June 1999

\begin{abstract}
This work completes the task of solving locally the Einstein-Ashtekar equations for degenerate data. The two remaining degenerate sectors of the classical $(3+1)$-dimensional theory are considered. First, with all densitized triad vectors linearly dependent and second, with only two independent ones. It is shown how to solve the Ashtekar-Einstein equations completely by suitable gauge fixing and choice of coordinates. Remarkably, the Hamiltonian weakly Poisson commutes with the conditions defining the sectors. The summary of degenerate solutions is given in an appendix.
\end{abstract}

PACS number: 0420

\section{Introduction}

Einstein's standard gravity theory corresponds to an open region in the real section of the Ashtekar theory phase space. The boundary $\dagger$ of that region is set up by degenerate data. There are several motivations to study the degenerate sector. First, a natural question which arises is whether or not the evolution could throw some data out of the Einstein theory region. However, then, since reality is preserved, the evolving data should cross the degenerate sector. Secondly, according to loop quantization, quantum excitations of the gravitational field are lower dimensional and define degenerate, non-invertible metric tensors (see [1]).

The degenerate data can be classified with respect to the rank of the densitized triad, and the rank of the squared triad (see the next section). It should be noted that all the considerations in this paper are local. Our classification of the degeneracy, in particular, applies only to open regions of the surface of initial data, whereas in a general case the types can vary from one region to another.

All the solutions of the Einstein-Ashtekar equations of types $(1,1)$ and $(2,2)$ were derived in $[6,7]$. In the first case [6], a general solution is the congruence of the integral curves defined by the triad and foliating $\Sigma$ which behave like $(1+1)$-dimensional vacuum spacetimes with a pair of massless complex-valued fields propagating along them. In the $(2,2)$ case [7], it was shown that the preservation of reality by the evolution implies the existence of a foliation of $\Sigma$ into the integral 2-surfaces tangent to a given triad. Analogously to Jacobson's case, the equations of $(3+1)$ gravity make the 2 -surfaces behave like $(2+1)$-dimensional empty spacetimes with an extra massless complex field assigned to each surface and propagating along it. An important observation was that the conditions defining each of the sectors Poisson commute with the Hamiltonian modulo themselves and the constraints.

$\dagger$ Meaning here just the closure of the region minus the region itself. 
In the present paper, the Einstein-Ashtekar equations will be solved for the remaining two types of degenerate data. In the first $(1,0)$ case the solution is spacetime which is a 'set of independently evolving points'. In the second $(2,1)$ case, the general solution is such that the surface of initial data $\Sigma$ is foliated by integral curves of the vector field from the triad. Nine complex fields evolve along these curves. As in the previously studied cases, it is shown that the conditions defining each degeneracy sector weakly (in the same sense as above) Poisson commute with the Hamiltonian $\dagger$.

Before the systematic study of the Ashtekar equations in the degenerate sector which was started by Jacobson [6], various aspects of the degenerate sector were discussed, for instance, by Jacobson and Romano [3], Bengtsson [2], Reisenberger [4] and Matschull [5] (see also more recent work [9]).

\section{Ashtekar's theory}

For the reader's convenience we shall briefly review Ashtekar's theory.

It is a canonical theory on a spacetime manifold $\Sigma \times \mathbb{R}$, where $\Sigma$ is a 3-real-surface of initial data (the 'space') and $\mathbb{R}$ is the one-dimensional space of values for a time parameter. The phase space consists of the pairs of fields $(A, E)$, where $A$ is an algebra $\operatorname{sl}(2, \mathbb{C})$-valued 1 -form on $\Sigma$ and $E$ is an $\operatorname{sl}(2, \mathbb{C})$-valued vector density field of weight 1 defined on $\Sigma$. Using local coordinates $\left(x^{a}\right)=\left(x^{1}, x^{2}, x^{3}\right)$ on $\Sigma$ and a basis $\left(\tau_{i}\right)=\left(\tau_{1}, \tau_{2}, \tau_{3}\right)$ of $\operatorname{sl}(2, \mathbb{C})$ we write

$$
A=A_{a}^{i} \tau_{i} \otimes \mathrm{d} x^{a}, \quad E=E^{i a} \tau_{i} \otimes \partial_{a},
$$

where $A_{a}^{i}, E^{i a}$ are complex-valued functions on $\Sigma$. We fix the standard bilinear complexvalued inner product in $\operatorname{sl}(2, \mathbb{C})$ by

$$
k(v, w):=-2 \operatorname{tr}(v w)
$$

for any $v, w \in \operatorname{sl}(2, \mathbb{C})$. The variables $(A, E)$ are canonically conjugate, the only nonvanishing Poisson bracket is

$$
\left\{A_{a}^{i}(x), E^{j b}(y)\right\}=\mathrm{i} k^{i j} \delta_{a}^{b} \delta(x, y) .
$$

Data $(A, E)$ are accompanied by Lagrange multipliers, a -1 weight density $N$ (the densitized lapse function), a vector field $N^{a}$ (the familiar shift) and an $\operatorname{sl}(2, \mathbb{C})$-valued function $\Lambda$, all defined on $\Sigma$. The Hamiltonian is given by

$$
\begin{aligned}
H & =\mathcal{C}_{N}+\mathcal{C}_{\vec{N}}+\mathcal{G}_{\Lambda}, \\
\mathcal{C}_{N} & :=\int_{\Sigma} \mathrm{d}^{3} x N \mathcal{C}(A, E):=-\frac{1}{2} \int_{\Sigma} \mathrm{d}^{3} x N F_{a b}^{i} E^{j a} E^{k b} c_{i j k}, \\
\mathcal{C}_{\vec{N}} & :=\int_{\Sigma} \mathrm{d}^{3} x N^{a} \mathcal{C}_{a}(A, E):=-\mathrm{i} \int_{\Sigma} \mathrm{d}^{3} x N^{a} F_{a b}^{i} E_{i}^{b}, \\
\mathcal{G}_{\Lambda} & :=\int_{\Sigma} \mathrm{d}^{3} x \Lambda_{i} \mathcal{G}^{i}(A, E):=\mathrm{i} \int_{\Sigma} \mathrm{d}^{3} x \Lambda_{i} D_{a} E^{i a},
\end{aligned}
$$

where

$$
F:=\frac{1}{2} F_{a b}^{i} \tau_{i} \otimes \mathrm{d} x^{a} \wedge \mathrm{d} x^{b}:=\mathrm{d} A+A \wedge A
$$

$\dagger$ Another interesting derivation of our result on the possibility of the evolution of non-degenerate data into degenerate data was given in [8]. 
is the curvature of $A$, and

$$
D_{a} w^{i}:=\partial_{a} w^{i}+c^{i}{ }_{j k} A_{a}^{j} w^{k}
$$

is the covariant derivative $\left(w^{i}\right.$ is a function on $\left.\Sigma\right) . c^{i}{ }_{j k}$ are the structure constants of $\operatorname{sl}(2, \mathbb{C})$ defined by

$$
\left[\tau_{i}, \tau_{j}\right]=c^{k}{ }_{i j} \tau_{k} .
$$

The constraints $\mathcal{C}_{N}, \mathcal{C}_{\vec{N}}, \mathcal{G}_{\Lambda}$ generate, respectively, the time evolution, diffeomorphisms of $\Sigma$ and the Yang-Mills gauge transformations

$$
\begin{aligned}
& A \longmapsto g^{-1} A g+g^{-1} \mathrm{~d} g, \\
& E \longmapsto g^{-1} E g,
\end{aligned}
$$

where $g$ is any $S L(2, \mathbb{C})$-valued function on $\Sigma$.

Apart from the resulting constraint equations, the data $(A, E)$ are subject to the following reality conditions:

$$
\begin{aligned}
& \operatorname{Im}\left(E^{i a} E_{i}^{b}\right)=0, \\
& \operatorname{Im}\left(\left\{E^{i a} E_{i}^{b}, \mathcal{C}_{N}\right\}\right)=0 .
\end{aligned}
$$

As long as the matrix $\left(E^{i a}\right)_{i, a=1,2,3}$ is of rank 3 and the signature of the symmetric matrix $\left(E^{i a} E_{i}^{b}\right)_{a, b=1,2,3}$ is $(+,+,+)$ one constructs $\mathrm{ADM}$ data from $(A, E)$ and the Ashtekar theory is equivalent to the Einstein gravity with the Lorentzian signature. However, the theory naturally extends to degenerate cases, when the ranks are lower than 3.

\section{Classification of degeneracies.}

Since the $E$ field is complex valued, in general the rank of the '2-area matrix' (see, e.g., [7]) $\left(E^{i a} E_{i}^{b}\right)$ is lower or equal to the rank of the $\left(E^{i a}\right)$ matrix. If we restrict ourselves to the semi-positive definite case of the 2-area matrix, the possible cases are $(0,0),(1,0),(1,1)$, $(2,1),(2,2)$ and $(3,3)$, where the numbers indicate the ranks of the triad matrix and the 2 -area matrix, respectively.

The examples of triad vector fields falling into specific sectors could be as follows:

$$
\begin{aligned}
& (0,0) \quad E=0, \quad(1,0) \quad E=\left(\tau_{1}+\mathrm{i} \tau_{2}\right) \otimes\left(\frac{\partial}{\partial x^{1}}\right), \quad(1,1) \quad E=\tau_{1} \otimes\left(\frac{\partial}{\partial x^{1}}\right), \\
& (2,1) \quad E=\left(\tau_{1}+\mathrm{i} \tau_{2}\right) \otimes\left(\frac{\partial}{\partial x^{1}}\right)+\tau_{3} \otimes\left(\frac{\partial}{\partial x^{3}}\right), \\
& (2,2) \quad E=\tau_{1} \otimes\left(\frac{\partial}{\partial x^{1}}\right)+\tau_{2} \otimes\left(\frac{\partial}{\partial x^{2}}\right), \\
& (3,3) \quad E=\tau_{1} \otimes\left(\frac{\partial}{\partial x^{1}}\right)+\tau_{2} \otimes\left(\frac{\partial}{\partial x^{2}}\right)+\tau_{3} \otimes\left(\frac{\partial}{\partial x^{3}}\right) .
\end{aligned}
$$

\section{Sector $(1,0)$}

Sector $(1,0)$ is defined as the one for which rank $\left(E^{i a}\right)=1, \operatorname{sign}\left(E^{i a} E_{i}^{b}\right)=(0,0,0)$ at the surface of initial data $\Sigma$. In this paragraph the Ashtekar equations for the sector $(1,0)$ will be solved. At the beginning, it is useful to choose a convenient gauge. One may show the following. 
Lemma 1.

$$
\begin{aligned}
{\left[\left(E^{i a} E_{i}^{b}=0\right)\right.} & \left.\wedge\left(\operatorname{rank}\left(E^{i a}\right)=1\right)\right] \\
& \Rightarrow\left[\exists g \in S L(2, \mathbb{C}): g^{-1} E g=\left(\tau_{1}+\mathrm{i} \tau_{2}\right) \otimes\left(E^{1 a} \partial_{a}\right)\right] .
\end{aligned}
$$

Proof. Let us assume that

$$
\begin{aligned}
& \operatorname{rank}\left(E^{i a}\right)=1, \\
& E^{i a} E_{i}^{b}=0 .
\end{aligned}
$$

Equality (15) implies that

$$
E=\lambda \tau_{1} \otimes E^{3}+\mu \tau_{2} \otimes E^{3}+\tau_{3} \otimes E^{3},
$$

where $\lambda, \mu$ are functions on $\Sigma$ and $E^{3}:=E^{3 a} \partial_{a} \neq 0$.

From (17) and (16) we conclude that

$$
1+\lambda^{2}+\mu^{2}=0
$$

By the fact given in the appendix we can make a gauge transformation such that $\operatorname{Im} \lambda=0$. It can be easily shown that we can transform $E$ with real $\lambda$ to

$$
E=\lambda^{\prime} \tau_{1} \otimes E^{3}+\mu \tau_{2} \otimes E^{3},
$$

with some new real function $\lambda^{\prime}$. It can be done using

$$
g=\left(\begin{array}{cc}
\cos \phi, & -\sin \phi \\
\sin \phi, & \cos \phi
\end{array}\right)
$$

with a suitably chosen $\phi \in \mathbb{R}$ (see the appendix).

From fact 1 it follows that

$$
\lambda^{\prime 2}+\mu^{2}=0
$$

hence $\mu= \pm \mathrm{i} \lambda^{\prime}$.

Our field variable takes now the simple form

$$
E=\lambda^{\prime}\left(\tau_{1} \pm i \tau_{2}\right) \otimes E^{3} .
$$

By another gauge, with

$$
g=\left(\begin{array}{cc}
\mathrm{i} & 0 \\
0 & -\mathrm{i}
\end{array}\right)
$$

we obtain the required form

$$
E=\left(\tau_{1}+\mathrm{i} \tau_{2}\right) \otimes E^{+},
$$

which ends the proof.

Now, let us change the basis in $s l(2, \mathbb{C})$ to $\left(\tau_{+}, \tau_{-}, \tau_{0}\right)$, where $\tau_{+}:=\tau_{1}+\mathrm{i} \tau_{2}, \tau_{-}:=\tau_{1}-\mathrm{i} \tau_{2}$, $\tau_{0}:=\tau_{3}$. The expression for the field $E$ takes the simple form

$$
E=\tau_{+} \otimes E^{+},
$$

where $E^{+}:=E^{+a} \partial_{a}=E^{1 a} \partial_{a}$. It is easy to calculate that in the new basis

$$
c_{+-0}=2 \mathrm{i}=c_{[+-0]} \text {, }
$$

and

$$
\left(k_{i j}\right)=\left(\begin{array}{lll}
0 & 2 & 0 \\
2 & 0 & 0 \\
0 & 0 & 1
\end{array}\right),
$$

where $i, j=+,-, 0$. 


\section{Constraints}

The constraint equations read now as follows:

$$
\begin{aligned}
& \mathcal{C} \equiv 0, \quad \mathcal{G}^{-} \equiv 0, \\
& \mathcal{C}_{a}=-2 \mathrm{i}\left(i\left(E^{+}\right) F^{-}\right)_{a}=0, \\
& \mathcal{G}^{0}=-2 i\left(E^{+}\right) A^{-}=0, \\
& \mathcal{G}^{+}=\mathrm{i} \partial_{a} E^{+a}+i\left(E^{+}\right) A^{0}=0,
\end{aligned}
$$

where $i$ denotes the inner product and we use the convention for $A^{-}, A^{0}$, to be defined analogously to $E^{+}$and $F^{-}:=\mathrm{d} A^{-}+(A \wedge A)^{-}$. We will also use this convention for the other components of the field variables.

Since $F^{-}=\mathrm{d} A^{-}-\mathrm{i} A^{-} \wedge A^{0}$, the following equality is true, provided the constraint equations are fulfilled:

$i\left(E^{+}\right)\left(\mathrm{d} A^{-} \wedge A^{-}\right)=i\left(E^{+}\right)\left(F^{-} \wedge A^{-}\right)=\left(i\left(E^{+}\right) F^{-}\right) \wedge A^{-}+F^{-}\left(i\left(E^{+}\right) A^{-}\right)=0$.

Hence the 3-form $\mathrm{d} A^{-} \wedge A^{-}=0$. Therefore, there exist coordinates on $\Sigma$ such that $A^{-}=\alpha \mathrm{d} \bar{z}$, where $\alpha$ is a function on $\Sigma$ and $\bar{z}=x-\mathrm{i} y(x, y$ are two of the three real coordinates on $\Sigma)$ or $\bar{z} \in \mathbb{R}$ (in this case $(x, y, \bar{z})$ are the real coordinates on $\Sigma$ ).

If $\alpha \neq 0$ we can make gauge transformation with $g=\mathrm{e}^{\mathrm{i} \lambda \tau_{3}}$, where $\lambda=-\log \alpha$. This gives $A^{-}=\mathrm{d} \bar{z}$ and leaves the form of $E$ unchanged. Indeed, let $g=\mathrm{e}^{\lambda \tau_{0}}$, with $\lambda$ any complex function on $\Sigma$. We know that $g^{-1}=\mathrm{e}^{-\lambda \tau_{0}}$. Therefore,

$$
\begin{aligned}
g^{-1} \tau_{ \pm} g & =\mathrm{e}^{-\lambda \tau_{0}} \tau_{ \pm} \mathrm{e}^{\lambda \tau_{0}}=\mathrm{e}^{-\lambda \tau_{0}} \tau_{ \pm}\left(1+\lambda \tau_{0}+\frac{1}{2} \lambda^{2} \tau_{0}^{2}+\cdots\right) \\
& =\mathrm{e}^{-\lambda \tau_{0}}\left(\tau_{ \pm}+\lambda \tau_{ \pm} \tau_{0}+\frac{1}{2} \lambda^{2} \tau_{ \pm} \tau_{0}^{2}+\cdots\right) \\
& =\mathrm{e}^{-\lambda \tau_{0}}\left(\tau_{ \pm}+\lambda \tau_{0} \tau_{ \pm} \mp \mathrm{i} \lambda \tau_{ \pm}+\frac{1}{2} \lambda^{2} \tau_{0} \tau_{ \pm} \tau_{0} \mp \frac{1}{2} \mathrm{i} \lambda^{2} \tau_{ \pm} \tau_{0}+\cdots\right) \\
& =\mathrm{e}^{-\lambda \tau_{0}}\left(\tau_{ \pm}+\lambda \tau_{0} \tau_{ \pm} \mp \mathrm{i} \lambda \tau_{ \pm}+\frac{1}{2} \lambda^{2} \tau_{0}^{2} \tau_{ \pm} \mp \mathrm{i} \lambda^{2} \tau_{0} \tau_{ \pm}+\frac{1}{2}(\mathrm{i} \lambda)^{2} \tau_{ \pm} \pm+\cdots\right) \\
& =\mathrm{e}^{-\lambda \tau_{0}} \mathrm{e}^{\lambda\left(\tau_{0} \mp \mathrm{i}\right)} \tau_{ \pm}=\mathrm{e}^{\mp \lambda \mathrm{i}} \tau_{ \pm}, \\
g^{-1} \tau_{0} g & =\tau_{0}, \\
g^{-1} \mathrm{~d} g & =\mathrm{e}^{-\lambda \tau_{0}} \mathrm{~d}\left(\mathrm{e}^{\lambda \tau_{0}}\right)=\mathrm{e}^{-\lambda \tau_{0}}\left((\mathrm{~d} \lambda) \tau_{0}+\frac{1}{2}\left(\mathrm{~d} \lambda^{2}\right) \tau_{0}^{2}+\cdots\right)=\mathrm{e}^{-\lambda \tau_{0}}(\mathrm{~d} \lambda) \tau_{0} \mathrm{e}^{\lambda \tau_{0}}=(\mathrm{d} \lambda) \tau_{0} .
\end{aligned}
$$

We will now solve the constraint equations separately for three possible cases.

(a) $A^{-}=\mathrm{d} \bar{z}, \bar{z}=x-\mathrm{i} y, x, y \in \mathbb{R}$.

It follows from (27) that

$$
E^{+}=E^{+z} \frac{\partial}{\partial z}+E^{+u} \frac{\partial}{\partial u}
$$

where $u \in \mathbb{R}, z=x+\mathrm{i} y$. Since $\mathrm{d} A^{-}=0$, from (26) and (27) we obtain

$$
i\left(E^{+}\right) A^{0}=0=\mathcal{G}^{+}-\mathrm{i} \partial_{a} E^{+a},
$$

hence we need to solve the equation

$$
\partial_{a} E^{+a}=0 .
$$

The general solution of this equation is

$$
E^{+a}=\varepsilon^{a b c} \Psi_{b, c},
$$

where $\Psi$ is any complex function on $\Sigma$. The condition $E^{+\bar{z}}=0$ gives $\Psi_{z}=\Phi_{, u}$ and $\Psi_{u}=\Phi_{, z}$ with some complex function $\Phi$. 
To solve the constraint equations completely we only have to consider the condition

$$
i\left(E^{+}\right) A^{0}=0 .
$$

This is a simple algebraic equation for $A^{0}$, provided $E^{+}$is fixed. To end this discussion, it should be noted that there are no constraints for $A^{+}$.

(b) $A^{-}=\mathrm{d} \bar{z}, \bar{z} \in \mathbb{R}$.

From (27) we obtain

$$
E^{+}=E^{+x} \frac{\partial}{\partial x}+E^{+y} \frac{\partial}{\partial y}
$$

with $(x, y, \bar{z})$ coordinates on $\Sigma$. It is easy to see that we can solve this case in the same way as we solved point (a). We should only exchange $z$ with $x$ and $u$ with $y$.

(c) $A^{-}=0$.

In this case $F^{-}=0$, hence $\mathcal{C}_{a} \equiv 0$. Moreover, $\mathcal{G}^{0} \equiv 0$. We only have to solve

$$
\partial_{a} E^{+a}=\mathrm{i} E^{+a} A_{a}^{0} \text {. }
$$

For any given $E^{+}$it is a simple equation for $A^{0}$. We can see that in this case we have no constraints on $E^{+}$and $A^{+}$.

\section{Evolution equations}

If we take the conditions $E^{-}=0, E^{0}=0$ and $A^{-}-\mathrm{d} \bar{z}=0$ as the additional constraints, it is easy to see that they weakly commute with the Hamiltonian so their vanishing is preserved by the time evolution provided the constraints are satisfied. In particular, the simple form of $E$ is preserved by the time evolution. In fact,

$$
\begin{aligned}
& \dot{E}^{-a}=-\mathrm{i}\left(c^{-}{ }_{-k} E^{-b}+c^{-}{ }_{0 k} E^{0 b}\right)\left(D_{b} E^{k a}\right)=0, \\
& \dot{E}^{0 a}=E^{+b}\left(\partial_{b} E^{-a}+\mathrm{i} A_{b}^{0} E^{-a}-\mathrm{i} A_{b}^{-} E^{0 a}\right)-E^{-b} D_{b} E^{+a}=0 .
\end{aligned}
$$

The gauge fixing $A^{-}=\mathrm{d} \bar{z}$ is also unchanged by the evolution. Namely,

$$
\dot{A}_{a}^{-}=E^{-a} F_{b a}^{0}-E^{0 a} F_{b a}^{-}=0 .
$$

The variable $E$ is independent of time:

$$
\dot{E}^{+a}=E^{+b}\left(\partial_{b} E^{0 a}+2 \mathrm{i} A_{b}^{-} E^{+a}-2 \mathrm{i} A_{b}^{+} E^{-a}\right)-E^{0 b} D_{b} E^{+a}=0 .
$$

Moreover,

$$
\dot{A}_{a}^{0}=2 E^{+b} F_{b a}^{-}-2 E^{-b} F_{b a}^{+}=0
$$

and

$$
\dot{A}_{a}^{+}=-E^{+b} F_{b a}^{0}+E^{0 b} F_{b a}^{+}=E^{+b}\left(\partial_{a} A_{b}^{0}-\partial_{b} A_{a}^{0}+2 \mathrm{i} A_{a}^{-} A_{b}^{+}\right) .
$$

In order to calculate all the above time derivatives we used constraint equations. We can show that the part of $A_{a}^{+}$tangential to $E^{+a}$ is independent of time and the transversal components are linear functions of time. In fact,

$$
\frac{\partial}{\partial t}\left(E^{+a} A_{a}^{+}\right)=E^{+a} \dot{A}_{a}^{+}=2 E^{+a} E^{+b} \partial_{[a} A_{b]}^{0}=0 .
$$

Hence the right-hand side of (37) is independent of time and $\frac{\partial}{\partial t} \dot{A}_{a}^{+}=0$. Now, it can be easily checked that the reality conditions are satisfied identically for the solutions of the constraint and the evolution equations. 


\section{Summary}

We have solved completely the $(1,0)$ sector of Ashtekar gravity. The general solution for this case (for a certain gauge fixing and choice of coordinates) is as follows. The fields $E^{-}$, $E^{0}$ vanish. The field $E^{+}$is given by (30) and vanishing of the component transversal to $A^{-}$ if $A^{-} \neq 0$ or $E^{+}$is arbitrary if $A^{-}=0 . A^{-}$is any closed 1 -form on $\Sigma, A^{0}$ is given by equation (31) and $A^{+}$is an arbitrary 1 -form. All the fields are constant in time except for $A^{+}$ which is constant in the direction of $E^{+}$and is linear in time in the other directions.

An interesting feature of these solutions is that after imposing certain initial constraints on the field variables at $t=t_{0}$, at each point they evolve independently of the other points. The points of $\Sigma$ 'cannot see each other during the evolution'.

\section{Sector $(2,1)$}

Sector $(2,1)$ is defined by $\operatorname{rank}\left(E^{i a}\right)=2$ and $\operatorname{sign}\left(E^{i a} E_{i}^{b}\right)=(+, 0,0)$ at $t=t_{0}$ (on the surface $\Sigma)$. The complete local solution of the Ashtekar-Einstein equations in the sector $(2,1)$ will be given in the present section. We will start from fixing a gauge freedom and a useful choice of coordinates.

\section{Lemma 2.}

$$
\begin{aligned}
{\left[\left(\operatorname{sign}\left(E^{i a} E_{i}^{b}\right)\right.\right.} & \left.=(+, 0,0)) \wedge\left(\operatorname{rank}\left(E^{i a}\right)=2\right)\right] \\
& \Rightarrow\left[\exists g \in S L(2, \mathbb{C}): \quad g^{-1} E g=\tau_{+} \otimes E^{+}+\tau_{0} \otimes E^{0} \quad \text { and } \quad A_{3}^{\prime 0}=0\right],
\end{aligned}
$$

where $A^{\prime} \stackrel{\text { def }}{=} g^{-1} A g+g^{-1} \mathrm{~d} g$ and $E^{0}$ is real.

Proof. We assume that

$$
\begin{aligned}
& \operatorname{rank}\left(E^{i a}\right)=2, \\
& \operatorname{sign}\left(E^{i a} E_{i}^{b}\right)=1 .
\end{aligned}
$$

Let us choose such a real basis $\left(e_{1}, e_{2}, e_{3}\right)$ in the tangent space to $\Sigma$ that $\left(E^{i a} E_{i}^{b}\right)=$ $\operatorname{diag}(0,0,1)$. From the fact in the appendix we conclude that there exists gauge transformation such that

$$
E=E^{k l} \tau_{k} \otimes e_{l}+\tau_{3} \otimes e_{3}
$$

where $k, l=1,2$.

The rank assumption (38) implies that $E^{2}=f E^{1}$, where $f$ is a complex function on $\Sigma$. Equation (39) gives $f= \pm \mathrm{i}$. The minus sign can be removed in the same way as in (21), which completes the proof.

From now on let us use the gauge given by the above lemma. We can make use of the reality of $E^{0}$ by choosing a convenient coordinate system $\left(x^{1}, x^{2}, x^{3}\right)$ such that

$$
E^{0}=\frac{\partial}{\partial x^{3}} .
$$




\section{Constraints}

Constraint equations now read as follows:

$$
\begin{aligned}
& \mathcal{C}=4 \mathrm{i} E^{+a} E^{0 b} F_{a b}^{-}=0, \\
& \mathcal{C}_{a}=E^{0 b} F_{a b}^{0}+2 E^{+b} F_{a b}^{-}=0, \\
& \mathcal{G}^{+}=\partial_{a} E^{+a}+\mathrm{i}\left(A_{a}^{+} E^{0 a}-A_{a}^{0} E^{+a}\right)=0, \\
& \mathcal{G}^{0}=\partial_{a} E^{0 a}+2 \mathrm{i} A_{a}^{-} E^{+a}=0, \\
& \mathcal{G}^{-}=-\mathrm{i} A_{a}^{-} E^{0 a}=0 .
\end{aligned}
$$

Due to (41), equation (46) is solved by $A_{3}^{-}=0$. Since $\partial_{a} E^{0 a}=0$, equation (45) is equivalent to $A_{a}^{-} E^{+a}=0$, or

$$
A_{1}^{-} E^{+1}=-A_{2}^{-} E^{+2}
$$

Equation (42) gives

$$
E^{+a} E^{0 b} F_{a b}^{-}=-E^{+1} \partial_{3} A_{1}^{-}-E^{+2} \partial_{3} A_{2}^{-}=0 .
$$

Let us assume that $E^{+2} \neq 0$. Because of (47) we have

$$
A_{1}^{-} \partial_{3} A_{2}^{-}=A_{2}^{-} \partial_{3} A_{1}^{-} \text {. }
$$

If we assume $A_{1}^{-} \neq 0$, this is equivalent to the condition that $A_{2}^{-}=\Omega A_{1}^{-}$, where $\Omega$ is a complex function on $\Sigma$ such that $\partial_{3} \Omega=0$. Thus

$$
A^{-}=A_{1}^{-}\left(\mathrm{d} x^{1}+\Omega\left(x^{1}, x^{2}\right) \mathrm{d} x^{2}\right)
$$

and

$$
E^{+}=-\Omega E^{+2} \frac{\partial}{\partial x^{1}}+E^{+2} \frac{\partial}{\partial x^{2}}+E^{+3} \frac{\partial}{\partial x^{3}} .
$$

We know, however, that the coordinates $x^{1}$ and $x^{2}$ can be chosen in such a way that instead of $\Omega$ we can put i (if $\operatorname{Im} \Omega \neq 0$ ) or 0 (if $\operatorname{Im} \Omega=0$ ). Let us assume then, that from now on $\Omega=0$ or $\Omega=\mathrm{i}$.

In order to solve the constraints completely we have to solve two more equations, namely (43) and (44). Straightforward calculation shows that

$$
E^{+b} F_{a b}^{-}=-E^{+b} \partial_{b} A_{a}^{-}-\mathrm{i} E^{+b} A_{b}^{0} A_{a}^{-},
$$

and

$$
E^{0 b} F_{a b}^{0}=-\partial_{3} A_{a}^{0}+2 \mathrm{i} A_{a}^{-} A_{3}^{+} .
$$

Hence (43) gives

$$
2 E^{+b} \partial_{b} A_{a}^{-}+\partial_{3} A_{a}^{0}=2 \mathrm{i} A_{a}^{-}\left(A_{3}^{+}-E^{+b} A_{b}^{0}\right) .
$$

Substituting (44) into the above equation gives

$$
\partial_{3} A_{a}^{0}=-2 \partial_{b}\left(E^{+b} A_{a}^{-}\right) .
$$

With a given $E^{+}$and $A^{-}$, the above equation describes the dependence of $A^{0}$ on the coordinate $x^{3}$. 
To end the analysis of the constraints we should add (44), which can be treated as the constraint on $A_{3}^{+}$, provided $E^{+}$and $A^{0}$ are known

$$
A_{3}^{+}=\mathrm{i} \partial_{a} E^{+a}+A_{a}^{0} E^{+a} \text {. }
$$

Finally, the case $E^{+1}=E^{+2}=0$ should be considered separately. However, the only difference in the family of solutions for this case is in the form of $A^{-}$. Now, we have no restrictions on $A_{1}^{-}$and $A_{2}^{-}$.

For $A_{1}^{-}=0$ we obtain from (47) that $A_{2}^{-}=0$ or $E^{+2}=0$, but these cases are included in the other ones.

Hence we have solved the constraint equations completely for the sector $(2,1)$.

\section{Evolution}

Let us now consider conditions $E^{-}=0, E^{0}-\partial / \partial x^{3}=0, A_{3}^{-}=0$ as the new additional constraints on the initial data. One can show that they commute weakly with the Hamiltonian, hence they are preserved by the evolution. In fact,

$\dot{E}^{-a}=E^{0 b} \partial_{b} E^{-a}+\mathrm{i} \mathcal{G}^{-} E^{0 a}+\mathrm{i} E^{0 b} A_{b}^{0} E^{-a}-E^{-b} D_{b} E^{0 a}=0$,

$\dot{E}^{0 a}=-2 E^{+b} \partial_{b} E^{-a}-E^{0 a} \partial_{b} E^{0 b}+\mathcal{G}^{0} E^{0 a}-2 \mathrm{i} E^{+b} A_{b}^{0} E^{-a}+2 E^{-b} D_{b} E^{+a}=0$,

$\dot{A}_{3}^{0}=2 E^{+b} F_{b 3}^{-}-2 E^{-b} F_{b 3}^{+}=E^{0 b} F_{3 b}^{0}=0$.

Moreover, due to constraint equations, we obtain

$$
\dot{A}_{a}^{-}=E^{-b} F_{b a}^{0}-E^{0 b} F_{b a}^{-}=F_{a 3}^{-},
$$

thus

$$
\begin{aligned}
& \dot{A}_{3}^{-}=0, \\
& \dot{A}_{1}^{-}=-\partial_{3} A_{1}^{-}, \\
& \dot{A}_{2}^{-}=-\partial_{3} A_{2}^{-} .
\end{aligned}
$$

In order to find the evolution of $E^{+}$, let us first calculate

$$
\dot{A}_{3}^{+}=-E^{+b} F_{b 3}^{0}+E^{0 b} F_{b 3}^{+}=-E^{0 a} E^{+b} F_{b a}^{0}=-E^{+b} \mathcal{C}_{b}=0 .
$$

Now we have

$$
\dot{E}^{+a}=-\mathrm{i} c^{+}{ }_{i j} E^{i b}\left(\partial_{b} E^{j a}+c^{j}{ }_{k l} A_{b}^{k} E^{l a}\right),
$$

thus

$$
\begin{gathered}
\dot{E}^{+a}=E^{+b} \partial_{b} E^{0 a}-E^{0 b} \partial_{b} E^{+a}-2 \mathrm{i} A_{b}^{+} E^{-b} E^{+a}+E^{+a} \mathcal{G}^{0}-2 \mathrm{i}\left(\partial_{b} E^{0 b}\right) E^{+a} \\
-\mathrm{i} E^{0 a} E^{0 b} A_{b}^{+}+\mathrm{i} E^{+a} E^{0 b} A_{b}^{0},
\end{gathered}
$$

and since the constraints show that $E^{0 b} A_{b}^{0}=A_{3}^{0}=0$, we obtain

$$
\dot{E}^{+a}=-\partial_{3} E^{+a}-\mathrm{i} E^{0 a} A_{3}^{+} .
$$

Since $E^{0 a} A_{3}^{+}$does not depend on time, equation (62) can be easily integrated for $E^{+a}(t)$.

We obtain similar equations for the components of $A^{0}$. In the same way as in (55) we find that $\dot{A}_{a}^{0}=F_{a 3}^{0}$, hence

$$
\dot{A}_{a}^{0}=-\partial_{3} A_{a}^{0}+2 \mathrm{i} A_{a}^{-} A_{3}^{+} .
$$

Again we have a simple linear equation for $A^{0}$. 
The last thing we need to do to solve the evolution equations completely is to find the function $A^{+}(t)$. Let us calculate

$$
\dot{A}_{a}^{+}=-E^{+b} F_{b a}^{0}+E^{0 b} F_{b a}^{+} .
$$

This gives

$$
\dot{A}_{a}^{+}=-2 E^{+b} \partial_{[b} A_{a]}^{0}-\mathcal{G}^{0} A_{a}^{+}+\left(\partial_{b} E^{0 b}\right) A_{a}^{+}+2 \mathrm{i} E^{+b} A_{b}^{+} A_{a}^{-}+2 E^{0 b} \partial_{[b} A_{a]}^{+}+2 \mathrm{i} E^{0 b} A_{[b}^{+} A_{a]}^{0} .
$$

Using the constraints we obtain

$$
\dot{A}_{a}^{+}=-2 E^{+b} \partial_{[b} A_{a]}^{0}+2 \mathrm{i} E^{+b} A_{b}^{+} A_{a}^{-}+2 \partial_{[3} A_{a]}^{+}+2 \mathrm{i} A_{[3}^{+} A_{a]}^{0},
$$

thus

$$
\dot{A}_{a}^{+}=\partial_{3} A_{a}^{+}+2 \mathrm{i} A_{a}^{-} E^{+b} A_{b}^{+}-\partial_{a} A_{3}^{+}+\mathrm{i} A_{a}^{0} A_{3}^{+}-2 E^{+b} \partial_{[b} A_{a]}^{0} .
$$

We can see that due to the second term on the right-hand side of the above equation $A_{1}^{+}$depends on $A_{2}^{+}$and vice versa. However, we can simplify this equation using the results of constraint analysis. Let us consider two different possibilities.

(a) $E^{+1}=E^{+2}=0$.

In this case $E^{+b} A_{b}^{+}=0$ and we obtain simple linear equations for $A_{1}^{+}$and $A_{2}^{+}$, namely

$$
\dot{A}_{a}^{+}=-\partial_{3} A_{a}^{+}-\partial_{a} A_{3}^{+}+\mathrm{i} A_{a}^{0} A_{3}^{+}-2 E^{+b} \partial_{[b} A_{a]}^{0} .
$$

(b) $E^{+2} \neq 0, E^{+1}=-\Omega E^{+2}(\Omega=0$ or i).

It is easy to calculate that

$$
\begin{aligned}
& \frac{\partial}{\partial t}\left(A_{2}^{+}-\Omega A_{1}^{+}\right)=\partial_{3}\left(A_{2}^{+}-\Omega A_{1}^{+}\right)-\left(\partial_{2}-\Omega \partial_{1}\right) A_{3}^{+}+\mathrm{i} A_{3}^{+}\left(A_{2}^{0}-\Omega A_{1}^{0}\right) \\
&-E^{+b}\left[\partial_{b}\left(A_{2}^{0}-\Omega A_{1}^{0}\right)-\left(\partial_{2}-\Omega \partial_{1}\right) A_{b}^{0}\right] .
\end{aligned}
$$

Hence we have a simple linear equation for $\left(A_{2}^{+}-\Omega A_{1}^{+}\right)(t)$. Substituting $\Omega A_{1}^{+}(t)+\left(A_{2}^{+}-\right.$ $\left.\Omega A_{1}^{+}\right)(t)$ for $A_{2}^{+}(t)$ in (66) we obtain the linear equation for $A_{1}^{+}(t)$. It can be integrated if $A^{-}, E^{+}, A_{3}^{+}, A^{0}$ are known.

This solves the evolution equations. One can see that the reality conditions are satisfied for all the solutions we have found.

\section{Summary}

Let us summarize the general solution of the Ashtekar-Einstein equations in the sector $(2,1)$. First, we have $E^{-}(t)=0$ and $E^{0}(t)=\partial / \partial x^{3}$. The fields $E^{+a}$ propagate along the integral curves of $E^{0}$ according to equation (62). The components $E^{+2}$ and $E^{+3}$ are arbitrary functions of the 'spatial' coordinates (but $E^{+3} \neq 0$ ) and the remaining component is given by $E^{+1}=-\Omega E^{+2}$, equation (50) ( $\Omega=0$ or i). If $E^{+2} \neq 0, A^{-}$is given by (49) with the same $\Omega$ as above, and if $E^{+2}=0$, arbitrary 1 -form $A^{-}$with $A_{3}^{-}=0$ is the solution. Fields $A_{1}^{-}$ and $A_{2}^{-}$propagate along the integral curves of $E^{0}$ at the speed of light. $A^{0}$ is any field which propagates along the same curves as $A^{-}$and $E^{+}$according to equation (63) and depends on the coordinate $x^{3}$ according to equation (51).

The field $A_{3}^{+}$does not depend on time and is given by equation (52). $A_{1}^{+}$and $A_{2}^{+}$are any functions on $\Sigma$ with the dependence on time given by (66).

It should be noted that, as in sector $(2,2)$, the characteristic feature of our solutions is the fact that evolution takes place on the curves, namely the curves defined by $x^{1}, x^{2}=$ constant. During the evolution these curves do not interact. 


\section{Concluding remarks}

As indicated in the introduction, all the possible degenerate sectors of Ashtekar's gravity have been solved. They all have certain important features in common.

First of all, the conditions defining the degeneracy sectors commute weakly with the Hamiltonian. Therefore, if $t=t_{0}$ corresponds to the surface of initial data $\Sigma$, then there is an $\varepsilon>0$ such that for all $t$ between $t_{0}$ and $t_{0}+\varepsilon$ the degeneracy type is the same (evolution preserves the degeneracy locally, where the word 'local' refers to both space and time). Hence if the initial data on $\Sigma$ are specified in such a way that all of them belong to the same degeneracy sector, the generic behaviour will be such that the evolution preserves the character of the degeneracy. On the other hand, if there are regions on $\Sigma$ with different types of data then the above need not be true (see [9]).

The other important feature is that for all the sectors, the surface of initial data $\Sigma$ is foliated by sub-manifolds of dimension equal to the rank of the densitized inverse 3-metric $q q^{a b}$ on $\Sigma$. The evolution always takes place in such a way that these sub-manifolds evolve independently. The time derivatives of the field variables on a fixed leaf of the foliation depend only on the values of these fields on the leaf and on the derivatives along the leaf. $q q^{a b}$ decides that the fields evolve along the surfaces [7], along the curves (sector $(2,1)$ and [6]), at the points independently (sector $(1,0))$ or do not evolve at all $\left(E^{i a}=0\right.$ for all $\left.i, a\right)$.

\section{Acknowledgments}

JL was supported by Alexander von Humboldt-Stiftung and the Polish Committee on Scientific Research (KBN, grant no 2 P03B 017 12). JW was supported by the Polish Ministry of Education and Stefan Batory Trust.

\section{Appendix}

Useful fact

Fact 1. Let $u^{i}, v^{i}, w^{i}$ be such vectors in $\operatorname{sl}(2, \mathbb{C})$ that $u^{i} u_{i}=v^{i} v_{i}=w^{i} w_{i}=1$ and $u^{i} v_{i}=u^{i} w_{i}=v^{i} w_{i}=0$. Then, there exists $g \in S L(2, \mathbb{C})$ such that

$$
g^{-1} u g=\tau_{1}, \quad g^{-1} v g=\tau_{2}, \quad g^{-1} w g=\tau_{3},
$$

where $\left(\tau_{i}\right)$ is an orthonormal basis in $\operatorname{sl}(2, \mathbb{C})$ such that $\left[\tau_{i}, \tau_{j}\right]=\varepsilon_{i j}{ }^{k} \tau_{k}$.

Proof. Let us fix

$\tau_{1}=\frac{1}{2}\left(\begin{array}{cc}0 & \mathrm{i} \\ \mathrm{i} & 0\end{array}\right), \quad \tau_{2}=\frac{1}{2}\left(\begin{array}{cc}\mathrm{i} & 0 \\ 0 & -\mathrm{i}\end{array}\right), \quad \tau_{3}=\frac{1}{2}\left(\begin{array}{cc}0 & 1 \\ -1 & 0\end{array}\right)$.

Let us check what transformation is made by

$$
g_{3}=\left(\begin{array}{cc}
\cos \phi & \mathrm{i} \sin \phi \\
\mathrm{i} \sin \phi & \cos \phi
\end{array}\right) \in S L(2, \mathbb{C})
$$

with $\phi \in \mathbb{R}$. It is easy to calculate that

$$
\begin{aligned}
& g_{3}^{-1} \tau_{1} g_{3}=\cos (2 \phi) \tau_{1}-\sin (2 \phi) \tau_{2}, \\
& g_{3}^{-1} \tau_{2} g_{3}=\sin (2 \phi) \tau_{1}+\cos (2 \phi) \tau_{2}, \\
& g_{3}^{-1} \tau_{3} g_{3}=\tau_{3} .
\end{aligned}
$$


Hence, choosing proper $\phi \in \mathbb{R}$ we can make any rotation around the $\tau_{3}$-axis in the vector space $\operatorname{sl}(2, \mathbb{C})$.

Analogously we can check that

$$
g_{1}=\left(\begin{array}{cc}
\mathrm{e}^{\mathrm{i} \phi} & 0 \\
0 & \mathrm{e}^{-\mathrm{i} \phi}
\end{array}\right) \quad \text { and } \quad g_{2}=\left(\begin{array}{cc}
\cos \phi & -\sin \phi \\
\sin \phi & \cos \phi
\end{array}\right)
$$

give rotations around the $\tau_{1}, \tau_{2}$ axes, respectively.

The above fact now follows from the properties of rotations in three-dimensional vector space.

\section{Complete set of local solutions for degenerate gravity.}

We shall list here the general solutions obtained for all possible kinds of degeneracy which can potentially occur in Ashtekar's theory for the Lorentzian signature. The interpretation and some properties of these solutions are given in the preceding sections.

Sector $(0,0) . \quad$ Where $E^{i a}=0, A_{a}^{i}$ are arbitrary, constant in time.

Sector $(1,0) . \quad E^{-a}=0, E^{0 a}=0$. There are two possibilities for $E^{+}$and $A^{-}$:

(a) $A_{a}^{-}=0, E^{+a}$ is arbitrary and constant in time, or

(b) $A_{a}^{-}=(\mathrm{d} \bar{z})_{a}(\bar{z}$ is a real or complex coordinate on $\Sigma), E^{+a}=\varepsilon^{a b c} \Psi_{b, c}$, where $\Psi_{a}$ is constant in time and $\Psi_{z}=\Phi_{, u}, \Psi_{u}=\Phi_{, z}$ with $\Phi$ an arbitrary function on $\Sigma$ and $z_{, z}=0$, $u_{, \bar{z}}=0$.

$A_{a}^{0}$ is given by $\partial_{a} E^{+a}=\mathrm{i} E^{+a} A_{a}^{0}$ and $A_{a}^{+}$is given by $\dot{A}_{a}^{+}=E^{+b}\left(\partial_{a} A_{b}^{0}-\partial_{b} A_{a}^{0}+2 \mathrm{i} A_{a}^{-} A_{b}^{+}\right)$.

$\operatorname{Sector}(1,1)$.

$$
E^{1 a}=E^{2 a}=0, \quad E^{3 a}=\left(\frac{\partial}{\partial x^{3}}\right)^{a}, \quad A_{3}^{i}=0,
$$

where $A_{a}^{3}$ is arbitrary, constant in time and $x^{3}, A_{a}^{1}, A_{a}^{2}$ are given by $\partial_{t}\left(A_{a}^{1} \pm \mathrm{i} A_{a}^{2}\right)=$ $\pm \partial_{3}\left(A_{a}^{1} \pm \mathrm{i} A_{a}^{2}\right)$.

Sector $(2,1)$.

$$
E^{-a}=0, \quad E^{0 a}=\left(\frac{\partial}{\partial x^{3}}\right)^{a},
$$

where $\left(E^{+2}, E^{+3}\right)$ are arbitrary functions of spatial coordinates $\left(E^{+3} \neq 0\right)$,

$$
E^{+1}=-\Omega E^{+2}
$$

$(\Omega=0$ or $\mathrm{i})$.

If $E^{+2} \neq 0$, then $A_{a}^{-}=A_{1}^{-}\left(\mathrm{d} x^{1}+\Omega \mathrm{d} x^{2}\right)_{a}$.

If $E^{+2}=0$, then $A_{a}^{-}$is an arbitrary function of spatial coordinates with $A_{3}^{-}=0$.

For both cases: $\dot{A}_{1}^{-}=-\partial_{3} A_{1}^{-}$and $\dot{A}_{2}^{-}=-\partial_{3} A_{2}^{-}$.

$A_{a}^{0}$ depends on spatial coordinates according to $\partial_{3} A_{a}^{0}=-2 \partial_{b}\left(E^{+b} A_{a}^{-}\right)$.

$A_{3}^{+}$is constant in time and given by $A_{3}^{+}=\mathrm{i} \partial_{a} E^{+a}+A_{a}^{0} E^{+a}$. 
Evolution of $A_{a}^{0}$ is given by $\dot{A}_{a}^{0}=-\partial_{3} A_{a}^{0}+2 \mathrm{i} A_{a}^{-} A_{3}^{+}$.

Evolution of $E^{+a}$ is determined from $\dot{E}^{+a}=-\partial_{3} E^{+a}-\mathrm{i} E^{0 a} A_{3}^{+}$.

$A_{1}^{+}$and $A_{2}^{+}$are arbitrary functions evolving according to

(a)

$$
\dot{A}_{a}^{+}=-\partial_{3} A_{a}^{+}-\partial_{a} A_{3}^{+}+\mathrm{i} A_{a}^{0} A_{3}^{+}-2 E^{+b} \partial_{[b} A_{a]}^{0},
$$

if $E^{+1}=E^{+2}=0$, or

$$
\begin{aligned}
& \frac{\partial}{\partial t}\left(A_{2}^{+} \pm \Omega A_{1}^{+}\right)=\partial_{3}\left(A_{2}^{+} \pm \Omega A_{1}^{+}\right)-\left(\partial_{2} \pm \Omega \partial_{1}\right) A_{3}^{+}+\mathrm{i} A_{3}^{+}\left(A_{2}^{0} \pm \Omega A_{1}^{0}\right) \\
& \quad-E^{+b}\left[\partial_{b}\left(A_{2}^{0} \pm \Omega A_{1}^{0}\right)-\left(\partial_{2} \pm \Omega \partial_{1}\right) A_{b}^{0}\right]
\end{aligned}
$$

if $E^{+2} \neq 0$.

Sector (2, 2).

$$
\begin{aligned}
& E^{1 a}=\left(\frac{\partial}{\partial x^{1}}\right)^{a}, \quad E^{2 a}=\left(\frac{\partial}{\partial x^{2}}\right)^{a}, \quad E^{3 a}=0, \\
& A_{1}^{i}=0, \quad A_{2}^{i}=0, \quad A_{3}^{1}=-\lambda_{, 2}, \quad A_{3}^{2}=\lambda_{, 1}, \quad A_{3}^{3}=\mathrm{i} \lambda_{, t},
\end{aligned}
$$

where $\lambda$ is any complex function satisfying $\lambda_{, t t}-\lambda, 11-\lambda, 22=0$.

\section{References}

[1] Ashtekar A 1991 Lectures on Non-perturbative Canonical Gravity (Singapore: World Scientific)

[2] Bengtsson I 1993 Some observations on degenerate metrics Gen. Rel. Grav. 25101

Bengtsson I 1990 A new phase for general relativity? Class. Quantum Grav. 727

[3] Jacobson T and Romano J D 1992 Degenerate extensions of general relativity Class. Quantum Grav. 9 L119

[4] Reisenberger M P 1995 New constraints for canonical general relativity Nucl. Phys. B $457643-87$

[5] Matschull H J 1996 Causal structure and diffeomorphisms in Ashtekar's gravity Class. Quantum Grav. 13 $765-82$

[6] Jacobson T $19961+1$ sector of $3+1$ gravity Class. Quantum Grav. 13 L111-6

[7] Lewandowski J and Wiśniewski J $19972+1$ sector of $3+1$ gravity Class. Quantum Grav. 14 775-82

[8] Ma Y and Liang C 1998 The degenerate sector of Ashtekar's phase space Mod. Phys. Lett. A $132839-43$

[9] Bengtsson I and Jacobson T 1997 Degenerate metric phase boundaries Class. Quantum Grav. 143109 